\title{
Article
}

\section{Levels of organochlorine pesticides are associated with amyloid aggregation in apex avian brains}

Heys, Kelly A, Shore, Richard F., Pereira, M. Gloria and Martin, Francis L

Available at https://clok.uclan.ac.uk/18580/

Heys, Kelly A, Shore, Richard F., Pereira, M. Gloria and Martin, Francis L orcid iconORCID: 0000-0001-8562-4944 (2017) Levels of organochlorine pesticides are associated with amyloid aggregation in apex avian brains. Environmental Science and Technology, 51 (15). pp. 8672-8681. ISSN 0013-936X

It is advisable to refer to the publisher's version if you intend to cite from the work. http://dx.doi.org/10.1021/acs.est.7b00840

For more information about UCLan's research in this area go to http://www.uclan.ac.uk/researchgroups/ and search for < name of research Group>.

For information about Research generally at UCLan please go to http://www.uclan.ac.uk/research/

All outputs in CLoK are protected by Intellectual Property Rights law, including Copyright law. Copyright, IPR and Moral Rights for the works on this site are retained by the individual authors and/or other copyright owners. Terms and conditions for use of this material are defined in the policies page.

\section{CLoK}

Central Lancashire online Knowledge www.clok.uclan.ac.uk

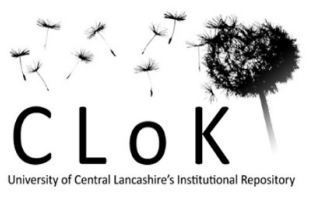




\title{
Levels of Organochlorine Pesticides Are Associated with Amyloid Aggregation in Apex Avian Brains
}

\author{
Kelly A. Heys, ${ }^{\dagger \neq}$ Richard F. Shore, ${ }^{\ddagger}$ M. Glória Pereira, ${ }^{\dagger}$ and Francis L. Martin ${ }^{* \dagger, \S_{\odot}}$ \\ ${ }^{\dagger}$ Lancaster Environment Centre, Lancaster University, Bailrigg, Lancaster LA1 4YQ U.K. \\ ${ }^{\ddagger}$ NERC Centre of Ecology and Hydrology, Lancaster Environment Centre, Lancaster LA1 4AP, U.K. \\ ${ }^{\S}$ School of Pharmacy and Biomedical Sciences, University of Central Lancashire, Preston, Lancashire, PR1 2HE, U.K.
}

\section{Supporting Information}

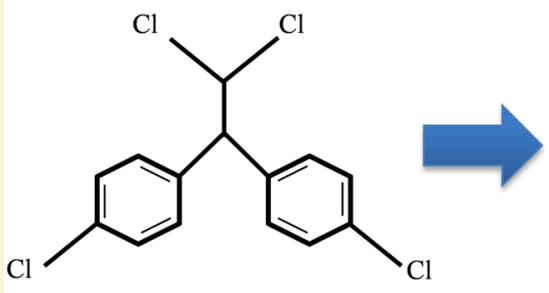

Organochlorine pesticides

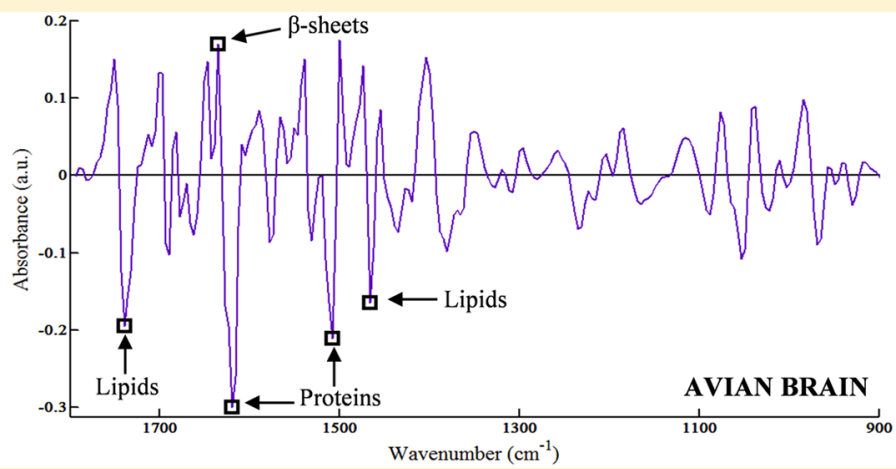

ABSTRACT: Organochlorine (OC) pesticides pose a significant environmental risk to wildlife and humans and have been associated with Alzheimer's disease $(\mathrm{AD})$. This study aims to spectroscopically analyze brains from free-flying birds and link the results to $\mathrm{OC}$ exposure and consequent amyloid aggregation. As long-lived apex predators, predatory birds represent a sentinel species similar to humans. Therefore, the results have implications for both species and may also add to our understanding of the role OC pesticides play in the development of $\mathrm{AD}$. Brains of wild Sparrowhawks were analyzed using ATR-FTIR and Raman spectroscopy and Congo red staining; results were correlated with OC pesticide concentrations in livers. Effects of OC exposure were sex- and age-dependent and associated alterations were seen in lipids and protein secondary structure. A shift from $\alpha$-helix to $\beta$-sheet conformation of proteins indicated that concentrations of OC pesticides $>7.18 \mu \mathrm{g} / \mathrm{g}$ may lead to cerebral amyloid aggregation.

\section{INTRODUCTION}

Organochlorine (OC) insecticides are a large and diverse class of compounds, many of which are highly lipophilic and persistent in the environment. They have attracted a lot of attention in the past due to widespread usage between the 1940s and 1970s and the ensuing recognition that some OCs have significant detrimental effects on the environment. ${ }^{1,2} \mathrm{OC}$ insecticides were banned in many countries during the 1990s due to environmental and human health concerns but are still used in many developing countries, particularly to control malaria. ${ }^{3}$ Since the global OC usage decreased due to substance control, there have been many questions regarding the longterm effects of environmental OC exposure. Even in the past three years, OC concentrations have been detected and measured in human blood samples from around the world ${ }^{4,5}$ as well as in many species of fish ${ }^{6,7}$ which is thought to represent a significant source of dietary OC intake. Recently, OCs have also been determined in environmental compartments including air, ${ }^{8}$ water, ${ }^{9}$ and soil ${ }^{10}$ and have been detected in various environmental species such as dolphins, ${ }^{10}$ bears ${ }^{11}$ and mussels. ${ }^{12}$ Such studies demonstrate that these persistent chemicals are still a current environmental concern, both for wildlife and for the human population.

OCs are known neurotoxins which is the mechanism by which they are able to control pest populations. They alter sodium and potassium channels, in particular causing persistent opening of sodium channels which allows constant firing of action potentials. ${ }^{13}$ The brain is particularly vulnerable to their effects as it is rich in lipids and has a low capacity for detoxification. Thus, OCs have been implicated in the development of neurodegenerative diseases including $\mathrm{Alz}$ heimer's disease $(\mathrm{AD})$ and Parkinson's disease (PD). $\mathrm{AD}$ patients have been reported to have higher serum levels of dichlorodiphenyldichloroethylene (DDE, a metabolite of DDT), while elevated levels of $\beta$-hexachlorocyclohexane have been found in patients with PD. ${ }^{14} \mathrm{AD}$ is characterized by the presence of aggregated amyloid- $\beta$ protein plaques as well as

Received: February 14, 2017

Revised: June 14, 2017

Accepted: June 21, 2017

Published: June 21, 2017 
neurofibrillary tangles composed of phosphorylated tau protein in the brain. ${ }^{15}$ The associated cognitive decline is caused by neuronal death and loss of synapses due to the presence of these protein aggregates. Many factors have been attributed to the causation of $\mathrm{AD}$ including genetic factors, aging and exposure to chemicals such as OCs. ${ }^{16}$ Environmental exposure to a number of chemicals, including OC pesticides, is thought to cause the aggregation of amyloid- $\beta$ proteins by inducing cellular oxidative stress. OCs may directly alter $\mathrm{NADH} / \mathrm{NAD}^{+}$ levels or interact with the respiratory chain in mitochondria leading to an increase in cellular reactive oxygen species (ROS) levels. ${ }^{17}$ This results in oxidation and aggregation of cellular proteins including amyloid- $\beta$.

$\mathrm{AD}$ is a major health issue in aging Western populations so research to further our understanding of the disease is essential. Although many studies aim to link chemical exposure with the etiology of $\mathrm{AD}$, investigations are often limited by availability of post-mortem brain tissue and environmental exposure data for those samples. ${ }^{18}$ In this study, we have obtained Sparrowhawk (Accipiter nisus) brain samples that have been analyzed for concentrations of various OC pesticides, (see Supporting Information (SI) Table S1) allowing for further analysis of potential OC-mediated amyloid aggregation. Birds of prey, such as sparrowhawks, are apex predators and occupy a niche at the top of their food chain in the same way that humans do. Also, free-flying predatory birds encounter cumulative, real world OC exposures making them ideal sentinels in which to study environmental pollutants. Many predatory bird species have been previously used as sentinel species to study environmental contaminant such as OCs, PBDEs, PCBs and heavy metals in tissue samples as well as using feathers, blood, and eggs. ${ }^{19,20}$ Using vibrational spectroscopy techniques that are able to identify alterations at the biomolecular level, we aim to determine cellular alterations caused by high and low OC exposures. In line with current thinking, we will also investigate whether exposure to $\mathrm{OC}$ pesticides can be linked with amyloid aggregation in Sparrowhawk brain samples using spectroscopy, staining and immunoassay methodologies. To our knowledge, this is a unique study that attempts to couple chemical exposure data and spectral data with cerebral amyloid aggregation in birds.

\section{MATERIALS AND METHODS}

Brain Samples. Sparrowhawk (Accipiter nisus) brain samples were obtained from the Predatory Bird Monitoring Scheme (http://pbms.ceh.ac.uk) which receives dead birds for analysis from members of the public in the UK. On receipt, the Sparrowhawk brains were removed from the carcasses and stored in a tissue archive at $-18{ }^{\circ} \mathrm{C}$. The brain samples for this study were chosen from the archived tissues from Sparrowhawks that died between 1979 and 1990 in the UK. The Sparrowhawk livers had been previously analyzed for wet weight $\mathrm{OC}$ pesticide concentrations using previously reported analysis methods and so the brain samples used were ranked according to the total OC pesticide concentrations found in the livers of the same birds. Total OC pesticide concentrations ranged from 0.56 to $82.31 \mu \mathrm{g} / \mathrm{g}$ in liver (see SI Table S1). Brain samples $(n=58)$ were ranked and selected so that there were two main sample groups; 30 with "high" total OC concentrations (15 male, 15 female) and 28 with "low" total OC concentrations ( 15 male, 13 female). Details of samples used are included in SI Table S1.
Spectral Signal of Amyloid Fibril. In order to investigate the spectral signal from amyloid, $A \beta 1: 42$ fibers were interrogated using Raman spectroscopy. Fifty $\mu \mathrm{M}$ of $\mathrm{A} \beta 1: 42$ was aggregated for 1 week in $10 \mathrm{mM}$ phosphate buffer and then spun down in an airfuge system (Beckman Coulter, (UK) High Wycombe, UK) for $1 \mathrm{~h}$ at $125000 \mathrm{~g}$ to pellet fibers. The pellet was resuspended in $100 \mu \mathrm{L}$ of distilled water and $10 \mu \mathrm{L}$ was deposited onto gold coated glass slides (Platypus Technologies, WI).

Spectroscopy Analysis of Amyloid Fibrils. For each sample, $1 \mathrm{~g}$ of brain material was spectroscopically analyzed on an infrared-reflective, low-E slide (Kevley Technologies, Chesterland, OH). For ATR-FTIR spectroscopy, five spectra were obtained per slide using a Bruker TENSOR 27 FTIR spectrometer with Helios ATR attachment containing a diamond IRE of $250 \mu \mathrm{m} \times 250 \mu \mathrm{m}$ (Bruker Optics, Coventry, UK). The ATR-FTIR was set to attain spectra with $8 \mathrm{~cm}^{-1}$ spectral resolution and 32 coadditions, allowing $3.84 \mathrm{~cm}^{-1}$ spectral data spacing. Mirror velocity was set to $2.2 \mathrm{kHz}$. After each sample was analyzed, the diamond was cleaned with distilled water and a new background was taken to account for environmental deviations. For Raman spectroscopy, samples were interrogated using an InVia Renishaw Raman spectrometer, containing a $785 \mathrm{~nm}$ excitation laser, coupled with a charge- coupled device (CCD) and Leica microscope systems (Leica Microsystems, Milton Keynes, UK). Before each session of taking spectra, the spectrometer was calibrated using a silicon source. Seven spectra were taken per sample using $100 \%$ laser power, $30 \mathrm{~s}$ exposure time and 2 accumulations with a 12001 $\mathrm{mm}^{-1}$ grating. Spectra of amyloid fibers were taken at $100 \%$ laser power, $35 \mathrm{~s}$ exposure time and three accumulations. Spectra were preprocessed and analyzed using the IRootLab toolbox (http://trevisanj.github.io/irootlab/) with Matlab 2013a (The Maths Works, MA). First, all spectra were cut to the fingerprint region of $900-1800 \mathrm{~cm}^{-1}$. ATR-FTIR spectra were preprocessed by baseline correction using second order differentiation, vector normalization and then mean centered. Spectral data from Raman were preprocessed in the same manner but spectra were wavelet denoised before mean centering. In order to extract features from the large spectral data set, principal component analysis (PCA) and linear discriminant analysis (LDA) were used. PCA was used as a data reduction technique and optimal number of PCs was input into LDA to minimize intraclass variation. Leave-one-out crosscalculation was employed to avoid overfitting data. Tentative wavenumber assignments were given to loadings using FTIR and Raman assignments reported. ${ }^{21,22}$ In order to investigate the secondary structure of proteins in brain samples from high and low $\mathrm{OC}$ concentration groups, deconvolution of the mean Amide I peak was performed using PeakFit v4.12 software (Systat Software Inc., San Jose, California). Using the software, spectra underwent baseline correction and were cut to the Amide I region of $1600-1700 \mathrm{~cm}^{-1}$. Second derivatives of spectra were used to identify subpeaks that were "hidden" within the Amide I peak. Final deconvolution of subpeaks was performed using Gaussian peak-fit functions with $20 \%$ smoothing. The $r^{2}$ value of each fitted curve was maintained above 0.999 .

Staining Amyloid Fibrils. All reagents were purchased from Sigma (Sigma-Aldrich, Dorset, UK) unless stated otherwise. A Congo red stock solution was made up in 100 $\mathrm{mL}$ of $80 \%$ ethanol using $0.3 \mathrm{~g}$ of Congo red and $0.3 \mathrm{~g} \mathrm{NaCl}$. This was diluted to a working solution with the addition of 1 
A
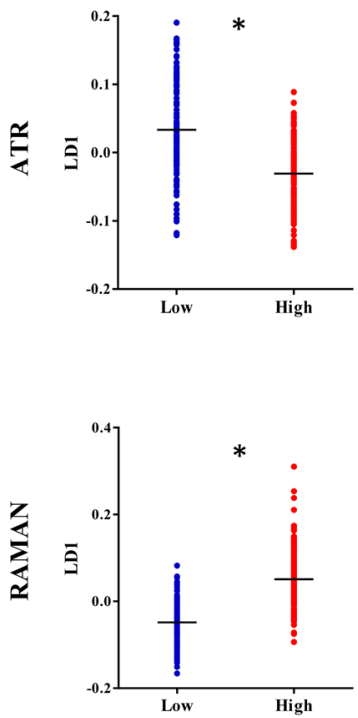

B
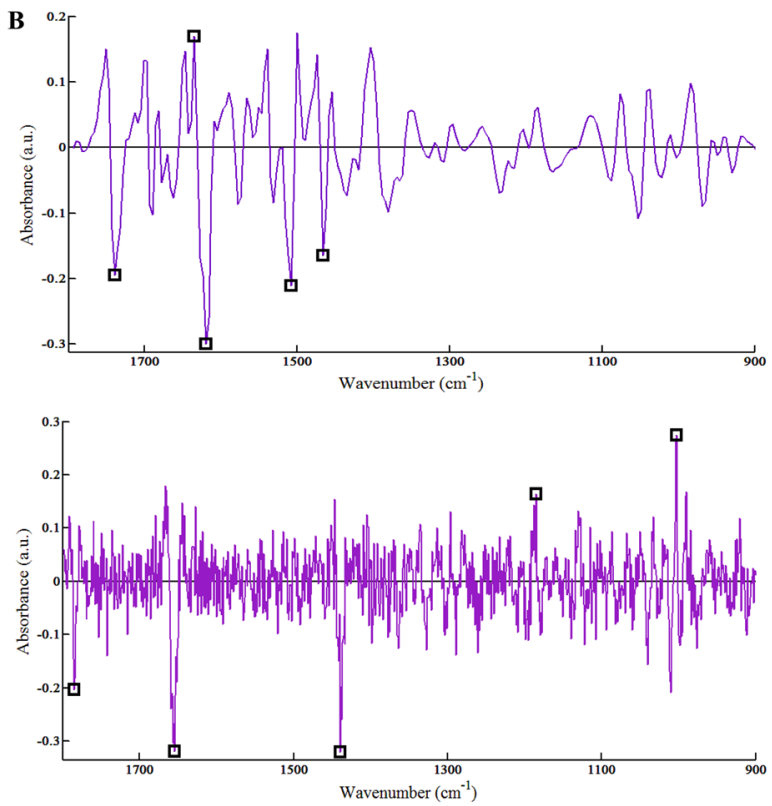

C

\begin{tabular}{|l|l|}
\hline $\begin{array}{l}\text { Wavenumber } \\
\left(\mathbf{c m}^{-1}\right)\end{array}$ & Assignment \\
\hline 1740 & $\mathrm{C}=\mathrm{O}$ stretching of lipids \\
\hline 1636 & Amide I of $\beta$ sheet structures \\
\hline 1620 & Amide I \\
\hline 1508 & Amide II \\
\hline 1466 & $\mathrm{CH}_{2}$ vibrations in lipids \\
\hline
\end{tabular}

\begin{tabular}{|l|l}
\hline $\begin{array}{l}\text { Wavenum } \\
\text { ber }\left(\mathbf{c m}^{-1}\right)\end{array}$ & Assignment \\
\hline 1785 & $\mathrm{C}=\mathrm{O}$ of lipids \\
\hline 1654 & Amide I of $\alpha$-helix structures \\
\hline 1440 & $\mathrm{CH}_{2}$ of lipids \\
\hline 1185 & Asymmetric phosphate \\
\hline 1003 & Phenylalanine \\
\hline
\end{tabular}

Figure 1. ATR-FTIR and Raman spectral comparison of brains samples from high and low OC exposure groups. (A) One-dimensional scores plots from cross-calculated PCA-LDA. Group means are represented by a black line and significance at the $P<0.01$ level as determined by unpaired, twotailed $t$ tests is indicated by an asterisk. (B) PCA-LDA loadings along LD1 with top five peaks highlighted and (C) Tentative biochemical assignments for the top five wavenumber peaks.

$\mathrm{mL}$ of $1 \% \mathrm{NaOH}$. For each sample, $1 \mathrm{~g}$ of brain material was transferred onto a glass microscope slide and stained with Congo red working solution for $15 \mathrm{~min}$. Slides were then rinsed in $\mathrm{dH}_{2} \mathrm{O}$ before differentiation in alkaline alcohol and counterstaining with hematoxylin. Following this, they were briefly exposed to blueing reagent and rinsed with tap water. Slides were viewed using a $15 \times$ Reflachromat objective on a Thermo Nicolet Continu $\mu \mathrm{m}$ microscope with cross-polarizing filters, fitted with an Olympus U-TV0.5XC-3 video camera. Microscopy image contrast was adjusted and then sharpened with a 0.55 weight Unsharp mask using ImageJ software (http://imagej.nih.gov/ij/).

Quantifying Amyloid A $1: 42$. To obtain tissue lysates suitable for ELISA, $100 \mathrm{mg}$ of brain tissue was homogenized, on ice, in $500 \mu \mathrm{L}$ of TBS with $1 \%$ triton X-100 and 2 mM EDTA. Tissue homogenates were then centrifuged for $20 \mathrm{~min}$ at 13000 $\mathrm{rpm}$. The supernatant was transferred to a fresh tube and stored at $-80{ }^{\circ} \mathrm{C}$. The ELISA was performed using a colorimetric BetaMark $\mathrm{x}$-42 ELISA kit from Biolegend (London, UK). The reagents, standards and test samples were diluted and prepared as outlined in the manufacturer's instructions. Fifty $\mu \mathrm{L}$ of sample was loaded into each well along with $50 \mu \mathrm{L}$ of horseradish peroxidase detection antibody and incubated overnight at $4{ }^{\circ} \mathrm{C}$. The next day, wells were washed five times and incubated, in the dark, with the tetramethylbenzidine substrate for $50 \mathrm{~min}$ at room temperature. The plate was read at $620 \mathrm{~nm}$ using a Tecan Infinite 200 Pro microplate reader (Tecan, Männedorf, Switzerland). Results were analyzed by construction of a 4PL standard curve and interpolation using Graphpad Prism 4.

Statistical Analyses. GraphPad Prism 4 (GraphPad Software Inc., CA) was used to carry out statistical tests unless otherwise stated. Two-tailed, unpaired $t$-tests in order to compare PCA-LDA scores from two sample groups (High vs Low) or to compare absorbance at specific peak locations in the case of CySS:Protein ratio and amyloid peak analysis. Two-way ANOVAs with Sidak multiple comparison tests were performed to compare PCA-LDA scores and account for interactions with other independent variables (Male vs Female or Adult vs Juvenile). T-tests and ANOVAs were done using sample spectral means rather than on individual spectra to avoid pseudoreplication. Normality of data was checked using D'Agostino-Pearson omnibus normality tests. Canonical correspondence analysis (CCA) was performed using XLSTAT (Addinsoft, New York). CCA is a direct gradient analysis technique and can be used to detect species variation patterns, which are caused by a provided set of environmental variables and thus explain variation in samples. Data was arranged into a species data table composed of spectral data and a table of environmental variables composed of OC concentration data. The CCA was run using 1000 random permutations.

\section{RESULTS}

Spectral Analysis of OC Exposed Brain Samples. To assess the effects of high and low OC pesticide exposure, Sparrowhawk brain samples were interrogated using ATR-FTIR and Raman spectroscopy and analyzed using cross-calculated PCA-LDA. High and low OC exposure groups were found to have significantly different spectral features. Figure 1A shows one-dimensional (1D) scores plots from PCA-LDA of ATRFTIR and Raman spectra which illustrate the difference between the two exposure groups along linear discriminant (LD) 1. In scores plots distance denotes dissimilarity, thus we can see that diverse spectral alterations were induced by low (below $2.03 \mu \mathrm{g} / \mathrm{g}$ ) and high (above $7.18 \mu \mathrm{g} / \mathrm{g}$ ) OC concentrations as demonstrated by the differences between group means and in distribution patterns. Unpaired, two-way $t$ tests using sample means (rather than spectral replicates) verified that the high and low exposure groups were significantly different at the $P<0.01$ level. Analysis of corresponding ATR and Raman LD1 loadings (Figure 1B) was performed to detect biospectral alterations that were responsible for the separation observed between groups in the 
ATR
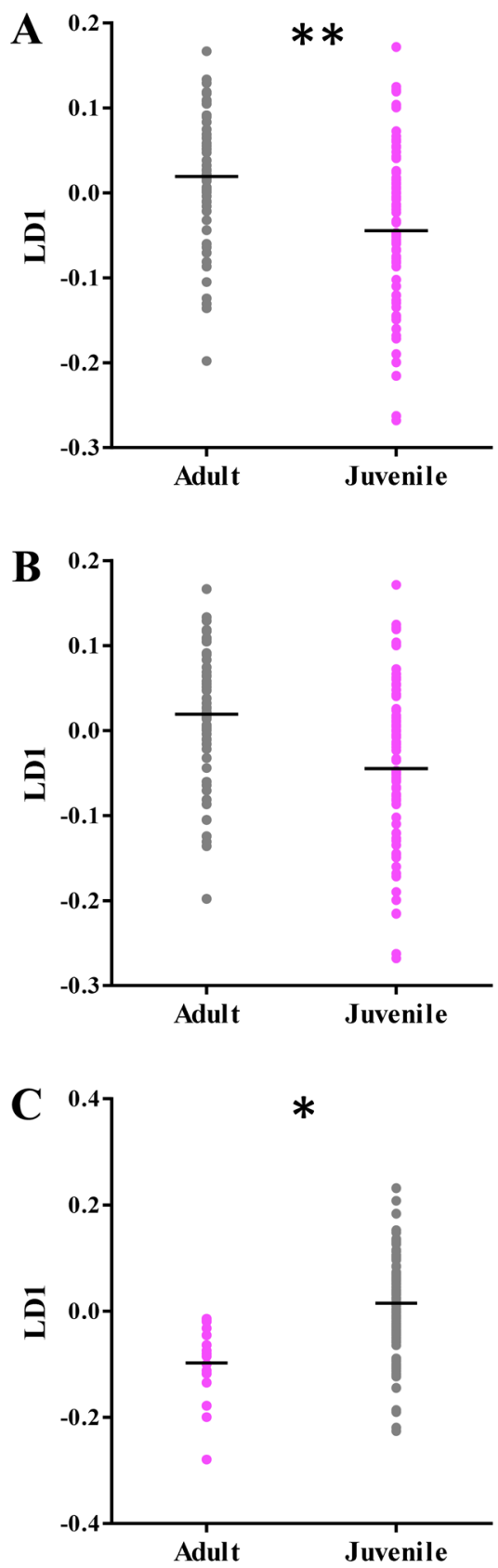

RAMAN
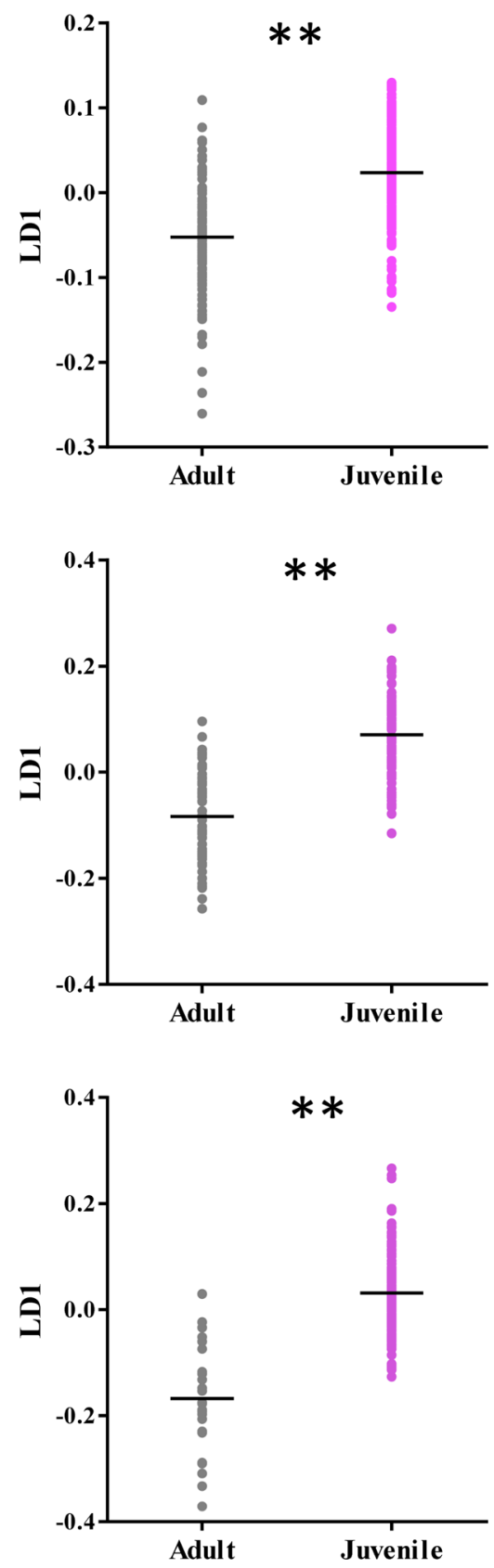

Figure 2. One-dimensional PCA-LDA scores plots from ATR-FTIR and Raman spectroscopy of brain samples showing age-dependent effects of OC exposure. (A) All samples, (B) high OC exposure group samples, and (C) low OC-exposure group samples. Group means are represented by a black line. Significance at the $P<0.05$ level as determined by two-way ANOVA with Sidak multiple comparison test is indicated by one asterisk and significance at the $P<0.01$ level is indicated by two asterisk.

scores plot. The top five peaks that contributed with the most magnitude to the observed variation were identified and tentative wavenumber alterations were assigned (Figure 1C). ATR-FTIR identified the top five wavenumber alterations in areas associated with $\mathrm{C}=\mathrm{O}$ stretching and $\mathrm{CH}_{2}$ vibrations of lipids $\left(1740 \mathrm{~cm}^{-1} ; 1466 \mathrm{~cm}^{-1}\right)$ and in regions associated with alterations in protein secondary structure. These comprised of changes in Amide I and Amide II spectral areas $\left(1620 \mathrm{~cm}^{-1}\right.$; $1508 \mathrm{~cm}^{-1}$ ) as well as alterations in $\beta$-sheet structures within the Amide I region $\left(1636 \mathrm{~cm}^{-1}\right)$. Wavenumber alterations detected by Raman spectroscopy confirmed that variation between the two exposure classes was due to alterations in $\mathrm{C}=$ $\mathrm{O}$ and $\mathrm{CH}_{2}$ lipid regions $\left(1785 \mathrm{~cm}^{-1} ; 1440 \mathrm{~cm}^{-1}\right)$ and spectral regions corresponding with protein secondary structure as changes in the $\alpha$-helix structures of Amide I $\left(1654 \mathrm{~cm}^{-1}\right)$ were detected. Raman spectral analysis also determined that alterations in asymmetric phosphate stretching vibrations from DNA $\left(1185 \mathrm{~cm}^{-1}\right)$ and the amino acid phenylalanine (1003 $\mathrm{cm}^{-1}$ ) contributed importantly to the difference between low and high OC pesticide exposed brain samples.

Information was available on the age and sex of the birds from which brain samples were obtained so spectra were 


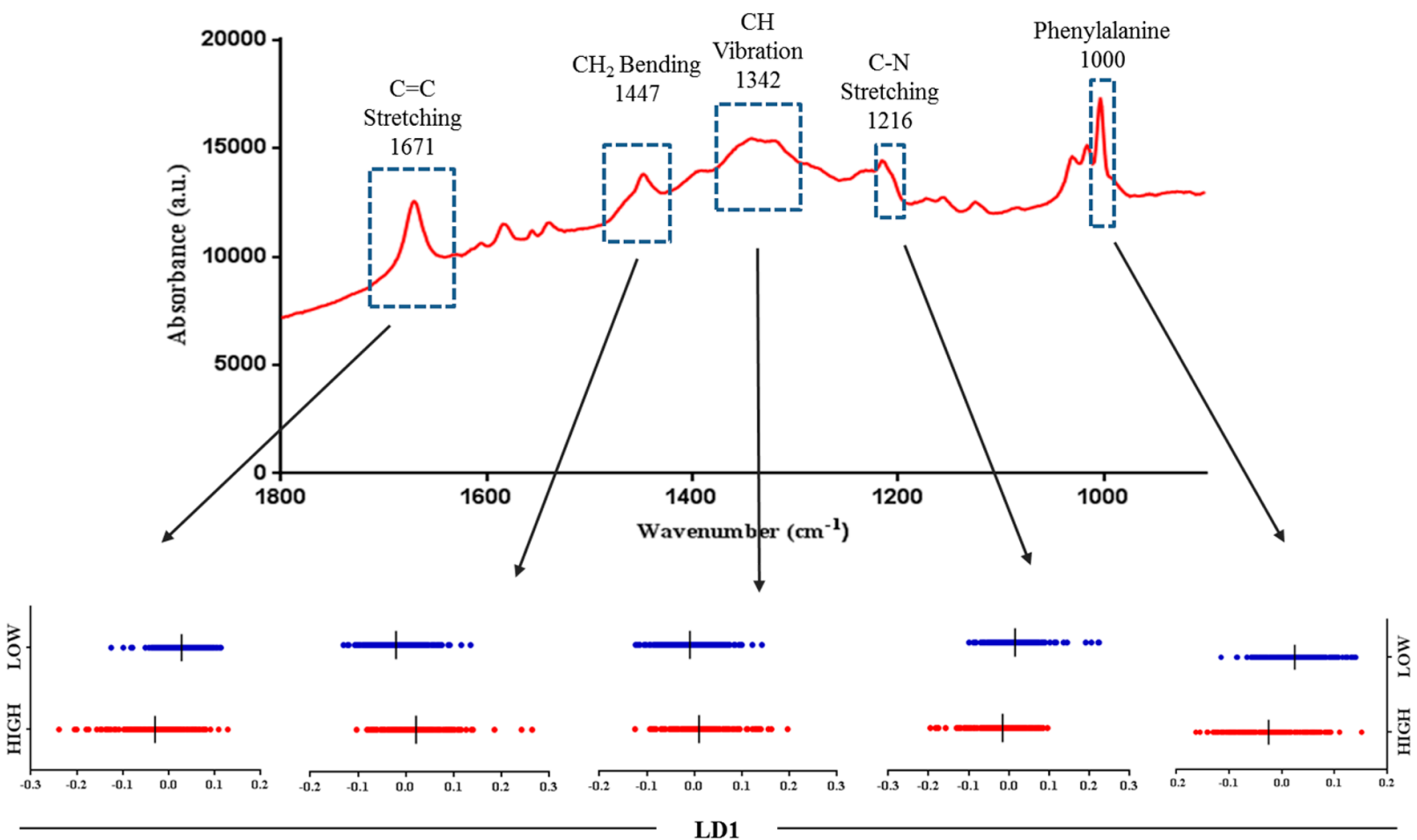

Figure 3. Raman spectra of amyloid A $\beta 1: 42$ fibrils with main vibrational peaks highlighted in blue. ID PCA-LDA scores plots underneath show separation of high and low OC-exposed brain samples at these vibrational nodes. Group scores are significantly different at the $P<0.05$ level as assessed by unpaired, two-way $t$ tests.

reclassified accordingly so that possible age- and sex-dependent effects of OC exposure could be investigated. The significance of differences between PCA-LDA scores along LD1 was assessed using two-way ANOVAs with Sidak multiple comparison tests in order to account for the other independent variables (sex, age, OC concentration). $P$-value results from these analyses can be seen in SI Table S2. ATR-FTIR and Raman PCA-LDA scores plots that were classed by sex (SI Figure S2A) show that there were significant $(P<0.01)$ differences between male and female scores. Group means are significantly separated and distribution of spectra is altered along LD1. Sex-dependent effects were also analyzed individually in high and low OC exposure groups. Raman scores from samples exposed to high OC concentrations were significantly separated by sex along LD1 at the $P<0.01$ level (SI Figure S2B). However, there was no significant difference found between male and female ATR-FTIR scores in the high OC group as there was a large amount of overlap between the two classes along the LD1 axis. Significant $(P<0.01)$ separation between male and female scores was revealed in the low OC exposure group using both spectral methods (SI Figure S2C). The effects of OC exposure were also found to be influenced by the age of the bird. Significant age-related differences at the $P<0.01$ level were found in both high and low OC exposure classes when analyzed with Raman and at the $P<0.05$ significance level for ATR-FTIR scores from the low OC exposure class (Figure $2 \mathrm{~B}$ and $\mathrm{C}$ ). Significant differences between adult and juvenile PCA-LDA scores were detected along LD1 using ATR-FTIR $(P<0.01)$ and Raman $(P<0.01)$ (Figure 3A). Therefore, the spectral results of exposure to OC pesticides are influenced by sex and age of the Sparrowhawk.
Contribution of Amyloid to Separation of High and Low OC-Exposed Samples. To investigate the involvement of amyloid in the difference observed between high and low OC-exposed brain samples, amyloid $\mathrm{A} \beta 1: 42$ fibrils were interrogated using Raman spectroscopy (Figure 3). The five major vibrational peaks were identified as $1671,1447,1342$, 1216 , and $1003 \mathrm{~cm}^{-1}$ which are wavenumbers associated with $\mathrm{C}=\mathrm{C}$ stretching, $\mathrm{CH}_{2}$ bending, $\mathrm{CH}$ deformation, $\mathrm{C}-\mathrm{N}$ stretching and phenylalanine, respectively. Some of these peaks are similar to those responsible for the separation of high and low Raman scores, in particular those related to regions of Amide $\mathrm{I}$ ( $\mathrm{C}=\mathrm{C}$ stretching), $\mathrm{CH}_{2}$ vibrations and phenylalanine. The Raman spectral results were analyzed to see if the two OC exposure groups were separated at wavenumbers associated with amyloid spectral peaks. It was found that scores from high and low OC exposure brains were significantly dissimilar $(P<0.05)$ along LD1 at all the major peaks found in the amyloid spectrum. To further investigate, the Amide I peak was deconvoluted to reveal "hidden" subpeaks (Figure 4A). Deconvolution of the Amide I peak from both high and low OC exposure classes resulted in five peaks related to secondary protein structure. Analysis of subpeaks from high OC exposure spectra showed that there was a lesser proportion of $\alpha$-helix than observed in the low OC exposure subpeaks. Conversely, more $\beta$-sheet was contributing to the Amide I peak in the high group than seen in the low group (see SI Table S3). As it is difficult to assess whether percentages are significantly different without additional values (numerator/denominator), we cannot state a definite significance but this should be explored in future work. 
A

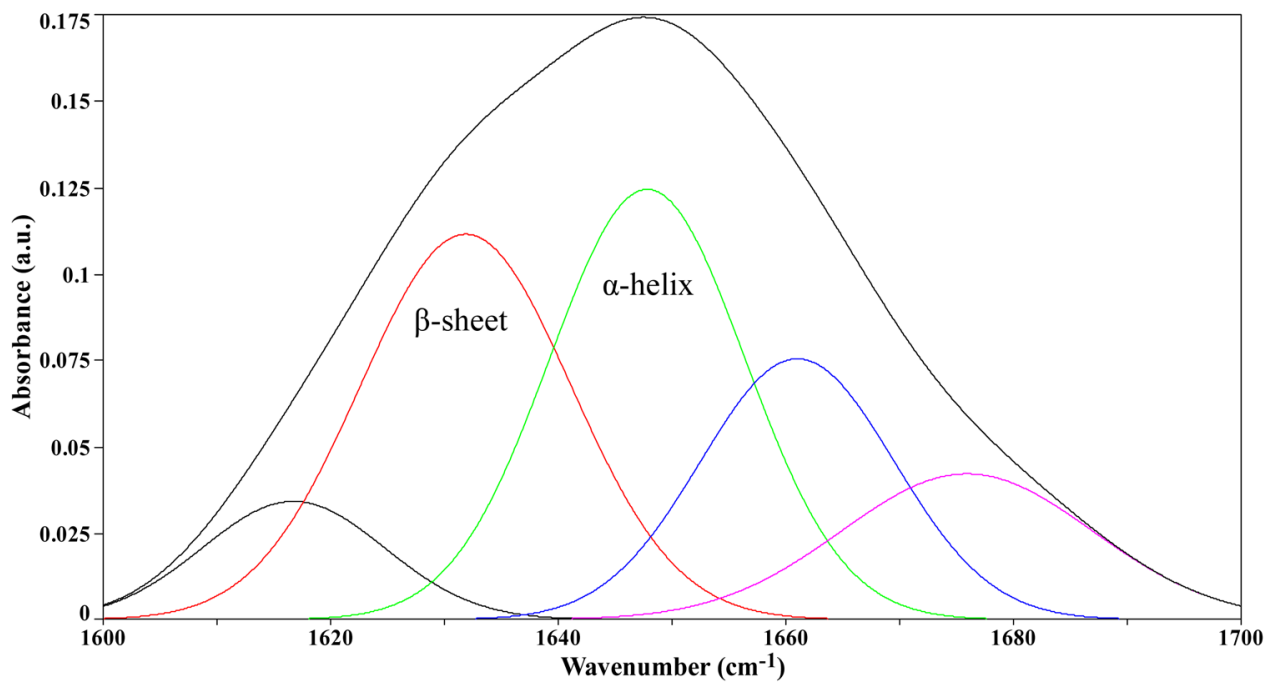

봅

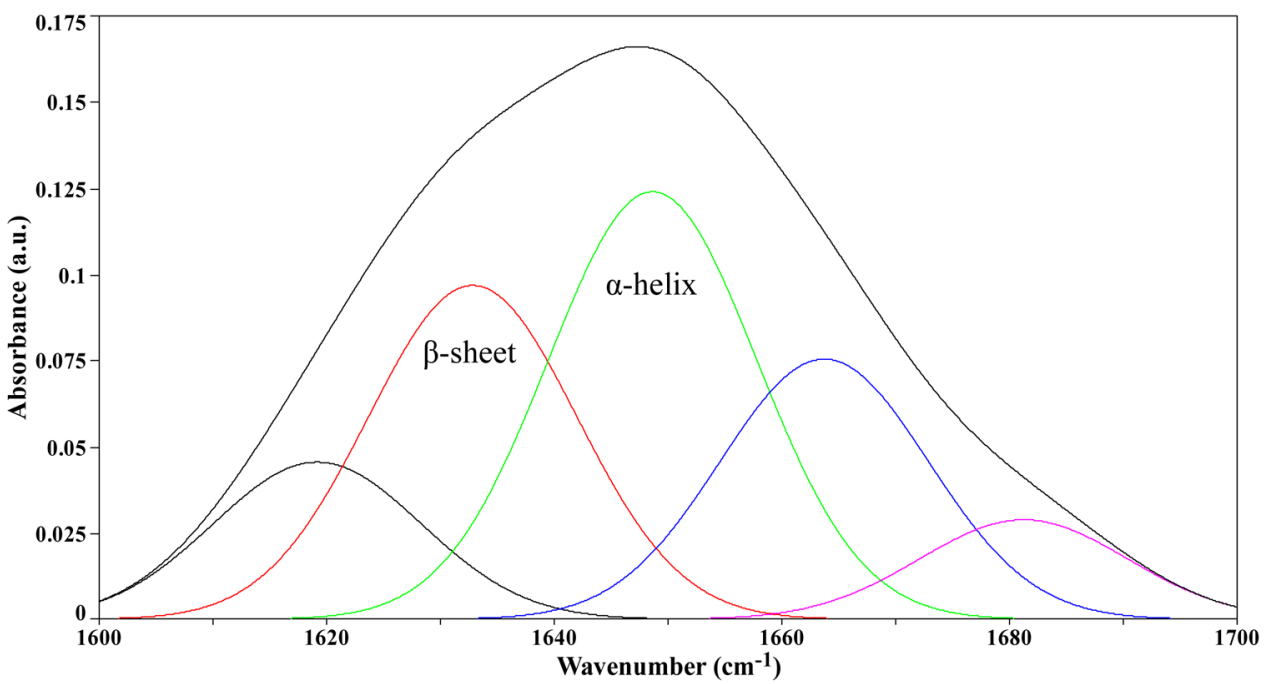

B
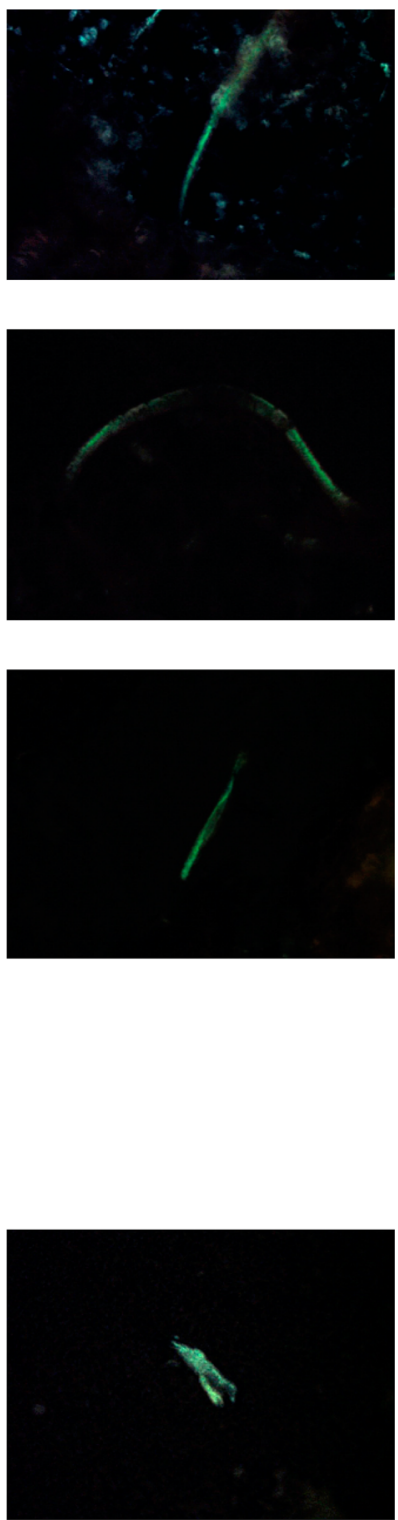

Figure 4. (A) Deconvolution of the Amide I peak derived from ATR-FTIR spectroscopy of high and low OC exposure groups, using second derivatives and Gaussian peak-fitting. (B) Microscopy images of Congo red stained brain samples, from high and low OC-exposure groups, which exhibited apple green birefringence when viewed under crossed polarizers.

Further to this, brain samples were stained with Congo red. All samples were stained and examined and four samples displayed the characteristic apple green color (Figure 4B). Three samples which were thin and fibrillar in appearance were from the high OC exposure group and one was from the low group. The three samples from the high group were all from male Sparrowhawks (two adults and one juvenile) and the sample from the low group was from a female. Finally, to confirm if amyloid was present in any of the samples, an ELISA was performed using lysates of the brain tissues (see SI Figure
S1). Amyloid A $\beta 1: 42$ was detected in two of the brain samples but the points did not fall within the linear portion of the standard curve and so quantification would not be accurate. The two samples which contained amyloid were both from the low OC exposure group and both from juvenile female birds.

Correlation of Spectral Results and OC Chemical Exposure. A CCA was performed to determine if total OC pesticide exposure was having an effect on the variation seen in the spectral results between the high and low OC exposed brain samples. The analysis showed that the constrained variables 


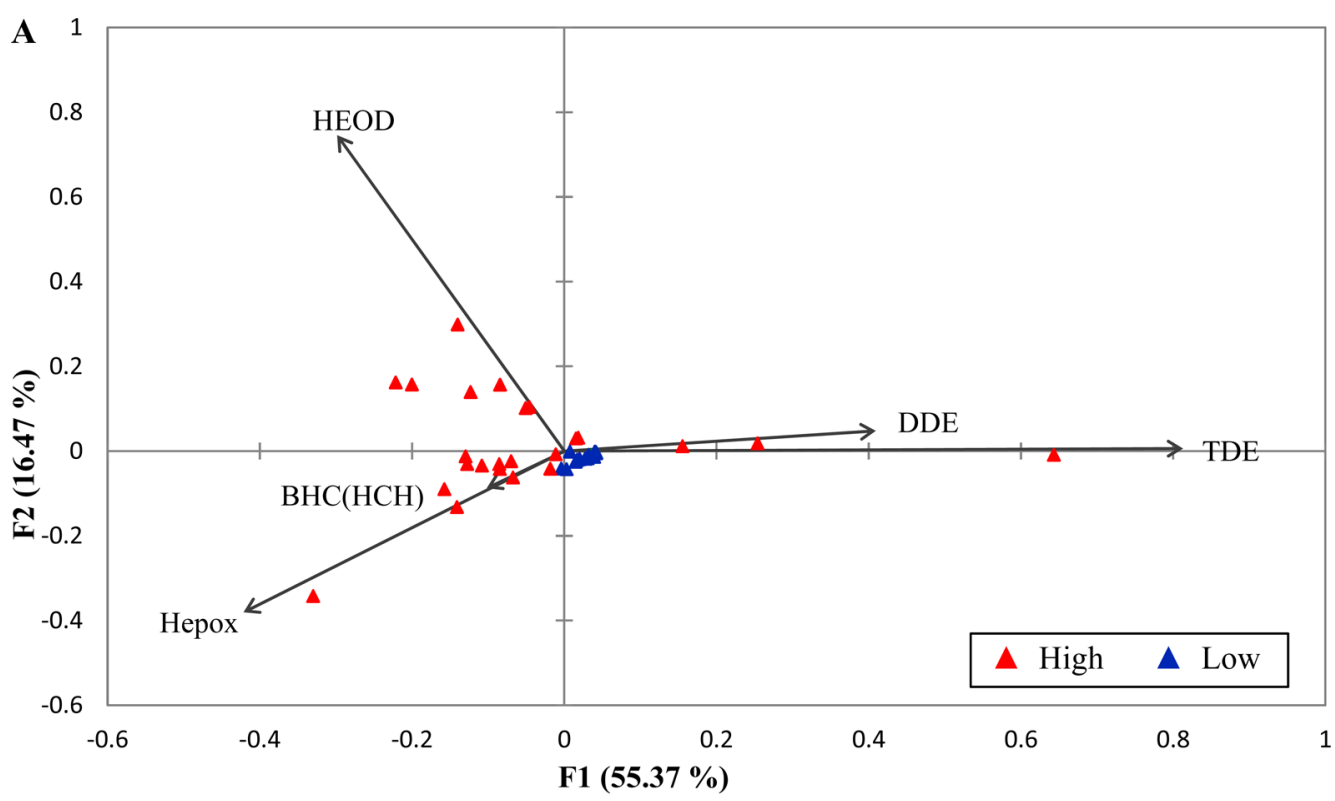

B

\begin{tabular}{|l|l|l|}
\hline Inertia & Value & $\begin{array}{l}\text { Percentage } \\
(\mathbf{\%})\end{array}$ \\
\hline Total & 0.301 & 100.000 \\
\hline Constrained & 0.037 & 12.185 \\
\hline Unconstrained & 0.264 & 87.815 \\
\hline
\end{tabular}

Figure 5. Correlation of chemical concentrations and ATR-FTIR spectral results from brain samples. (A) CCA ordination plot and (B) Inertia (variation) table showing correlation of spectral and chemical data from brain samples. Abbreviations as follows: $\mathrm{BHC}(\mathrm{HCH})$, $(\beta)$ Hexachlorocyclohexane; TDE, tetrachlorodiphenylethane; Hepox, Heptachlor epoxide; DDE, dichlorodiphenyldichloroethylene; HEOD, Dieldrin.

explained $12.185 \%$ of the inertia observed (Figure 5B). This means that the imputed variables, that is, the chemical OC concentration data explain $12 \%$ of the variation seen in the spectral data. The remaining variance is due to other uncontrolled factors. The ordination plot (Figure 5A) showed that points from the low $\mathrm{OC}$ exposure group are more strongly coclustered than those from the high group, which exhibit more scattering and variability. There is a small amount of overlap but largely, the two groups are well separated and clustered along the F1 axis. Assessment of the length and position of chemical directional arrows shows that TDE, heptachlor epoxide and HEOD are the OC pesticides that are most important and influential for the ordination. It also shows that DDE and TDE exposures are correlated as are $\mathrm{BHC}(\mathrm{HCH})$ and heptachlor epoxide exposures.

\section{DISCUSSION}

The category of vibrational spectroscopy is composed of many techniques including FTIR and Raman methodologies which allow fast yet detailed analysis of biological samples. Such techniques are powerful tools which can be used to reveal alterations in biochemical composition and structure at the molecular level. ${ }^{23}$ Infrared spectroscopy has previously been used in the monitoring and interrogation of environmental samples including assessment of the effect of water quality on tadpoles, ${ }^{24}$ identifying biomarkers of water contamination in English sole ${ }^{25}$ and detection of chemical exposure profiles in
Egret feathers to use as a monitoring tool. ${ }^{26}$ Here, we have used ATR-FTIR and Raman spectroscopy to assess the effects of OC pesticide exposure and the implication of such exposures to amyloid aggregation in Sparrowhawk brains. Computational analysis of spectra was carried out to allow examination of spectral scores and loadings. We have identified that the major wavenumber associated alterations that occur in response to exposure to $\mathrm{OC}$ pesticides arise in lipids and the secondary structure of proteins. This is consistent with what we understand about the toxic mechanism of OC pesticides which can induce alterations in a range of biomolecules including proteins and lipids by modifying endocrine and apoptotic pathways. ${ }^{27}$ The toxicity of strongly lipophilic chemicals such as OCs is often enhanced in the brain which is lipid rich and has poor detoxification mechanisms. As modifications in lipids and the secondary structure of proteins were identified by both ATR and Raman, these alterations may represent spectral markers of OC pesticide exposure in avian brain tissue.

The spectral results of OC pesticide exposure were found to be influenced by the sex and age of the bird. Sex-related differences in the body burdens of OC chemicals as well as other contaminants have previously been reported. ${ }^{28}$ Research suggests that female body concentrations may be lower than their male counterparts due to loss of body mass during breeding and through the maternal transfer of chemicals into eggs. ${ }^{29}$ The reported figures regarding the amount that is passed into eggs vary greatly but it is thought that as much as a 
third of a female's OC burden could be transferred in this way and incorporated into the lipid-containing yolk. ${ }^{30}$ Maternal transfer is also a relevant consideration for humans as secretion of OC pesticides into the breast milk of mammals including humans has been reported. ${ }^{31,32}$ The impact of age on the consequences of OC pesticide exposure has been well studied due to reports of neurodegeneration in aged individuals who are known to have been occupationally exposed to OCs during their lifetime. ${ }^{33,34}$

Exposure to OC pesticides is regarded as an important environmental risk factor in the development of $\mathrm{AD} .^{35}$ This study investigates the presence of amyloid in wild avian brains and attempts to link this to "real world" OC pesticide exposures. Using ATR-FTIR and Raman spectroscopy, we have identified that alterations in proteins, primarily changes in $\alpha$-helix and $\beta$-sheet content, are induced by exposure to OCs. This suggests that a significant modification in secondary protein structure occurs in response to elevated OC pesticide concentrations (those above $7.18 \mu \mathrm{g} / \mathrm{g}$ ). This is typically observed in amyloid- $\beta$ protein aggregation which is characterized by a shift from $\alpha$-helix to $\beta$-sheet formation as the peptide changes to an insoluble form. ${ }^{36}$ Congo red staining also indicated that amyloid was present in four brain samples, three of which were exposed to high concentrations of OCs. This again suggests that exposure to higher concentrations of such pesticides may lead to amyloid aggregation in brain tissue. An exploratory $\mathrm{A} \beta 1: 42$ ELISA detected the peptide in two brain samples. Interestingly, the two birds from which these samples came from were both juvenile females and were both from the low OC pesticide exposure group. This suggests that other factors are also important in the process leading to amyloid aggregation and that possibly there are genetic factors involved. In humans, an increase in cerebral $A \beta 1: 42$ has been associated with early onset $\mathrm{AD} .{ }^{37}$ As proposed by some "two-hit" hypotheses, genetic influences may act as a predisposition to amyloid aggregation that is mediated by other factors such as pesticide exposure. There are, however, many other factors involved in disease progression such as smoking, brain trauma and diet. ${ }^{38}$ As there are numerous implicated factors, a CCA was performed to understand the relationship between the $\mathrm{OC}$ pesticide exposure and spectral results from the brain samples. This established that $12 \%$ of spectral variation was due to the influence of OCs, which is a reasonable amount as environmental and spectral data sets are complex with multiple factors to consider. In conjunction with spectral analysis, this lends evidence to the argument that exposure to elevated levels of OC pesticides are implicated in the aggregation of amyloid and possibly in the development of $\mathrm{AD}$. Multiple studies have also found elevated $\mathrm{OC}$ pesticide levels in humans with $\mathrm{AD} .^{14,39}$

Despite large scientific and financial input, there is currently no cure for $\mathrm{AD}$ and we still do not fully understand many aspects of the disease. ${ }^{40}$ It is therefore imperative that research efforts continue to further our understanding of $\mathrm{AD}$. However, many studies focusing on the role of chemical contaminants are often limited by the availably of chemical data and brain tissue from the same test subjects. Studies such as this one, aim to increase our knowledge base of the etiology of $\mathrm{AD}$ as predatory birds occupy a very similar position in the food chain as humans do. Being apex predators and relatively long-lived makes birds of prey ideal surrogates in lieu of human experimental subjects. The use of tissue and data from wild free flying birds, via initiatives such as the PBMS, allows us to study the effects of "real world" exposure profiles. Recent reports have also suggested that the brains of birds are more similar to those of humans than previously thought and so the avian brain has been recognized as a potential model for human neuroscience. ${ }^{41,42}$ The results of studies like this may also be significant for avian research as well. Although wild birds do not usually live as long as their captive counterparts, many predatory bird species such as eagles and owls are remarkably long-lived and experience similar chemical exposures. If there are genetic predisposing factors as there are in humans, then younger birds and those with shorter lifespans may also be vulnerable to amyloid aggregation. Previously, an amyloid plaque has been identified in the brain of an aged captive woodpecker so there is a precedent of amyloid aggregation in the avian brain. ${ }^{43}$ This also demonstrates that if birds live long enough, aggregation of the amyloid protein into cerebral plaques is possible. In homing pigeons, neurodegeneration due to age related synaptic loss has been associated with reduced spatial cognitive function. ${ }^{44}$ If predatory birds develop amyloid aggregations and suffer synaptic loss, as has been demonstrated in humans, this could affect navigation and hunting behaviors, which require spatial cognition. Predatory birds may represent a new opportunity to study amyloid aggregation and $\mathrm{AD}$ risk factors, which has significant research implications for both humans and wildlife. This study also demonstrates that vibrational spectroscopy could be a useful tool in the investigation of $\mathrm{OC}$ pesticide exposure and potentially to identify markers of amyloid aggregation in brain tissue.

\section{ASSOCIATED CONTENT}

\section{S Supporting Information}

The Supporting Information is available free of charge on the ACS Publications website at DOI: 10.1021/acs.est.7b00840.

Results that show findings of human antiamyloid $\mathrm{A} \beta 1: 42$ calorimetric ELISA assay on Sparrowhawk brain lysates, sex-dependent effects of OC exposure, the panel of chemicals measured as wet weight concentration of contaminant in liver, and significance tests of PCA-LDA LD1 scores with age and sex (PDF)

\section{AUTHOR INFORMATION}

\section{Corresponding Author}

*Phone: +44-1771-896482; e-mail: flmartin@uclan.ac.uk.

\section{ORCID}

Francis L. Martin: 0000-0001-8562-4944

Notes

The authors declare no competing financial interest.

\section{ACKNOWLEDGMENTS}

Kelly Heys is a NERC algorithm student. We are grateful to Dr Claire Tinker-Mill for providing the amyloid fibril sample for analysis.

\section{REFERENCES}

(1) Walker, C. H.; Sibly, R. M., Hopkin, S. P.; Peakall, D. B. Principals of Ecotoxicology. CRC Press, 2012.

(2) Blus, L. J.; Neely, B. S.; Belisle, A. A.; Prouty, R. M. Organochlorine residues in brown pelican eggs: relation to reproductive success. Environ. Pollut. (1970-1979) 1974, 7 (2), 81-91.

(3) Ali, U.; Syed, J. H.; Malik, R. N.; Katsoyiannis, A.; Li, J.; Zhang, G.; Jones, K. C. Organochlorine pesticides (OCPs) in South Asian region: a review. Sci. Total Environ. 2014, 476, 705-717. 
(4) Wang, H.-S.; Chen, Z.-J.; Wei, W.; Man, Y.-B.; Giesy, J. P.; Du, J.; Zhang, G.; Wong, C. K.-C.; Wong, M.-H. Concentrations of organochlorine pesticides (OCPs) in human blood plasma from Hong Kong: Markers of exposure and sources from fish. Environ. Int. 2013, 54, 18-25.

(5) Lam, T.; Williams, P. L.; Lee, M. M.; Korrick, S. A.; Birnbaum, L. S.; Burns, J. S.; Sergeyev, O.; Revich, B.; Altshul, L. M.; Patterson, D. G., Jr Prepubertal serum concentrations of organochlorine pesticides and age at sexual maturity in Russian boys. Environ. Health Perspect. 2015, 123 (11), 1216.

(6) Yohannes, Y. B.; Ikenaka, Y.; Saengtienchai, A.; Watanabe, K. P.; Nakayama, S. M.; Ishizuka, M. Concentrations and human health risk assessment of organochlorine pesticides in edible fish species from a Rift Valley lake-Lake Ziway, Ethiopia. Ecotoxicol. Environ. Saf. 2014, 106, 95-101.

(7) Ameur, W. B.; Trabelsi, S.; El Megdiche, Y.; Hassine, S. B.; Barhoumi, B.; Hammami, B.; Eljarrat, E.; Barceló, D.; Driss, M. R. Concentration of polychlorinated biphenyls and organochlorine pesticides in mullet (Mugil cephalus) and sea bass (Dicentrarchus labrax) from Bizerte Lagoon (Northern Tunisia). Chemosphere 2013, 90 (9), 2372-2380.

(8) Meire, R. O.; Khairy, M.; Targino, A. C.; Galvão, P. M. A.; Torres, J. P. M.; Malm, O.; Lohmann, R. Use of passive samplers to detect organochlorine pesticides in air and water at wetland mountain region sites (S-SE Brazil). Chemosphere 2016, 144, 2175-2182.

(9) Temoka, C.; Wang, J.; Bi, Y.; Deyerling, D.; Pfister, G.; Henkelmann, B.; Schramm, K.-W. Concentrations and mass fluxes estimation of organochlorine pesticides in Three Gorges Reservoir with virtual organisms using in situ PRC-based sampling rate. Chemosphere 2016, 144, 1521-1529.

(10) Arienzo, M.; Albanese, S.; Lima, A.; Cannatelli, C.; Aliberti, F.; Cicotti, F.; Qi, S.; De Vivo, B. Assessment of the concentrations of polycyclic aromatic hydrocarbons and organochlorine pesticides in soils from the Sarno River basin, Italy, and ecotoxicological survey by Daphnia magna. Environ. Monit. Assess. 2015, 187 (2), 1-14.

(11) Romanić, S. H.; Klinčić, D.; Kljaković-Gašpić, Z.; Kusak, J.; Reljić, S.; Huber, Đ. Organochlorine pesticides and polychlorinated biphenyl congeners in wild terrestrial mammals from Croatia: Interspecies comparison of residue levels and compositions. Chemosphere 2015, 137, 52-58.

(12) Galvao, P.; Henkelmann, B.; Longo, R.; Dorneles, P. R.; Torres, J. P. M.; Malm, O.; Schramm, K.-W. Partition of organochlorine concentrations among suspended solids, sediments and brown mussel Perna perna, in tropical bays. Chemosphere 2014, 114, 9-15.

(13) O’Reilly, A. O.; Khambay, B. P.; Williamson, M. S.; Field, L. M.; Wallace, B.; Davies, T. E. Modelling insecticide-binding sites in the voltage-gated sodium channel. Biochem. J. 2006, 396 (2), 255-263.

(14) Richardson, J. R.; Roy, A.; Shalat, S. L.; von Stein, R. T.; Hossain, M. M.; Buckley, B.; Gearing, M.; Levey, A. I.; German, D. C. Elevated serum pesticide levels and risk for Alzheimer disease. JAMA neurology 2014, 71 (3), 284-290.

(15) Perl, D. P. Neuropathology of Alzheimer's disease. Mt. Sinai J. Med. 2010, 77 (1), 32-42.

(16) Manivannan, Y.; Manivannan, B.; Beach, T. G.; Halden, R. U. Role of Environmental Contaminants in the Etiology of Alzheimer's Disease: A Review. Curr. Alzheimer Res. 2015, 12 (2), 116.

(17) Thany, S. H.; Reynier, P.; Lenaers, G. [Neurotoxicity of pesticides: its relationship with neurodegenerative diseases]. Medecine sciences: $M / S$ 2013, 29 (3), 273-278.

(18) Zaganas, I.; Kapetanaki, S.; Mastorodemos, V.; Kanavouras, K.; Colosio, C.; Wilks, M. F.; Tsatsakis, A. M. Linking pesticide exposure and dementia: What is the evidence? Toxicology 2013, 307, 3-11.

(19) Newton, I.; Wyllie, I.; Asher, A. Long-term trends in organochlorine and mercury residues in some predatory birds in Britain. Environ. Pollut. 1993, 79 (2), 143-151.

(20) Gómez-Ramírez, P.; Shore, R.; Van den Brink, N.; Van Hattum, B.; Bustnes, J.; Duke, G.; Fritsch, C.; García-Fernández, A.; Helander, B.; Jaspers, V. An overview of existing raptor contaminant monitoring activities in Europe. Environ. Int. 2014, 67, 12-21.
(21) Movasaghi, Z.; Rehman, S.; ur Rehman, D. I. Fourier transform infrared (FTIR) spectroscopy of biological tissues. Appl. Spectrosc. Rev. 2008, 43 (2), 134-179.

(22) Movasaghi, Z.; Rehman, S.; Rehman, I. U. Raman spectroscopy of biological tissues. Appl. Spectrosc. Rev. 2007, 42 (5), 493-541.

(23) Baker, M. J.; Trevisan, J.; Bassan, P.; Bhargava, R.; Butler, H. J.; Dorling, K. M.; Fielden, P. R.; Fogarty, S. W.; Fullwood, N. J.; Heys, K. A. Using Fourier transform IR spectroscopy to analyze biological materials. Nat. Protoc. 2014, 9 (8), 1771-1791.

(24) Strong, R. J.; Halsall, C. J.; Ferenčík, M.; Jones, K. C.; Shore, R. F.; Martin, F. L. Biospectroscopy reveals the effect of varying water quality on tadpole tissues of the common frog (Rana temporaria). Environ. Pollut. 2016, 213, 322-337.

(25) Malins, D. C.; Anderson, K. M.; Stegeman, J. J.; Jaruga, P.; Green, V. M.; Gilman, N. K.; Dizdaroglu, M. Biomarkers signal contaminant effects on the organs of English sole (Parophrys vetulus) from Puget Sound. Environ. Health Perspect. 2006, 114, 823-829.

(26) Llabjani, V.; Malik, R. N.; Trevisan, J.; Hoti, V.; Ukpebor, J.; Shinwari, Z. K.; Moeckel, C.; Jones, K. C.; Shore, R. F.; Martin, F. L. Alterations in the infrared spectral signature of avian feathers reflect potential chemical exposure: A pilot study comparing two sites in Pakistan. Environ. Int. 2012, 48, 39-46.

(27) Mrema, E. J.; Rubino, F. M.; Brambilla, G.; Moretto, A.; Tsatsakis, A. M.; Colosio, C. Persistent organochlorinated pesticides and mechanisms of their toxicity. Toxicology 2013, 307, 74-88.

(28) Robinson, S. A.; Lajeunesse, M. J.; Forbes, M. R. Sex differences in mercury contamination of birds: testing multiple hypotheses with meta-analysis. Environ. Sci. Technol. 2012, 46 (13), 7094-7101.

(29) Zheng, X.-B.; Luo, X.-J.; Zeng, Y.-H.; Wu, J.-P.; Mai, B.-X. Chiral polychlorinated biphenyls (PCBs) in bioaccumulation, maternal transfer, and embryo development of chicken. Environ. Sci. Technol. 2015, 49 (2), 785-791.

(30) Newton, I.; Bogan, J.; Marquiss, M. Organochlorine contamination and age in sparrowhawks. Environ. Pollut., Ser. A 1981, 25 (2), 155-160.

(31) Cadieux, M. A.; Muir, D. C.; Béland, P.; Hickie, B. E. Lactational transfer of polychlorinated-biphenyls (PCBs) and other organochlorines in St. Lawrence beluga whales (Delphinapterus leucas). Arch. Environ. Contam. Toxicol. 2016, 70 (1), 169-179.

(32) Chávez-Almazán, L. A.; Diaz-Ortiz, J.; Alarcón-Romero, M.; Davila-Vazquez, G.; Saldarriaga-Noreña, H.; Sampedro-Rosas, L.; López-Silva, S.; Santiago-Moreno, A.; Rosas-Acevedo, J. L.; Waliszewski, S. M. Influence of Breastfeeding Time on Levels of Organochlorine Pesticides in Human Milk of a Mexican Population. Bull. Environ. Contam. Toxicol. 2016, 96 (2), 168-172.

(33) Hayden, K. M.; Norton, M. C.; Darcey, D.; Østbye, T.; Zandi, P. P.; Breitner, J.; Welsh-Bohmer, K.; Investigators, C. C. S.. Occupational exposure to pesticides increases the risk of incident $\mathrm{AD}$ The Cache County Study. Neurology 2010, 74 (19), 1524-1530.

(34) Kamel, F.; Tanner, C.; Umbach, D.; Hoppin, J.; Alavanja, M.; Blair, A.; Comyns, K.; Goldman, S.; Korell, M.; Langston, J. Pesticide exposure and self-reported Parkinson's disease in the agricultural health study. Am. J. Epidemiol. 2007, 165 (4), 364-374.

(35) Ballard, C.; Gauthier, S.; Corbett, A.; Brayne, C.; Aarsland, D.; Jones, E., Alzheimer's disease. Lancet 2011377, (9770), 10191031.10.1016/S0140-6736(10)61349-9

(36) Kotler, S. A.; Walsh, P.; Brender, J. R.; Ramamoorthy, A. Differences between amyloid- $\beta$ aggregation in solution and on the membrane: insights into elucidation of the mechanistic details of Alzheimer's disease. Chem. Soc. Rev. 2014, 43 (19), 6692-6700.

(37) Lista, S.; Garaci, F. G.; Ewers, M.; Teipel, S.; Zetterberg, H.; Blennow, K.; Hampel, H. CSF A $\beta 1-42$ combined with neuroimaging biomarkers in the early detection, diagnosis and prediction of Alzheimer's disease. Alzheimer's Dementia 2014, 10 (3), 381-392.

(38) Reitz, C.; Mayeux, R. Alzheimer disease: epidemiology, diagnostic criteria, risk factors and biomarkers. Biochem. Pharmacol. 2014, 88 (4), 640-651. 
(39) Singh, N.; Chhillar, N.; Banerjee, B.; Bala, K.; Basu, M.; Mustafa, M. Organochlorine pesticide levels and risk of Alzheimer's disease in north Indian population. Hum. Exp. Toxicol. 2013, 32 (1), 24-30.

(40) Association, A. s. 2016 Alzheimer's disease facts and figures. Alzheimer's Dementia 2016, 12 (4), 459-509.

(41) Chen, C. C.; Winkler, C. M.; Pfenning, A. R.; Jarvis, E. D. Molecular profiling of the developing avian telencephalon: regional timing and brain subdivision continuities. J. Comp. Neurol. 2013, 521 (16), 3666-3701.

(42) Clayton, N. S.; Emery, N. J. Avian models for human cognitive neuroscience: a proposal. Neuron 2015, 86 (6), 1330-1342.

(43) Nakayama, H.; Katayama, K.-I.; Ikawa, A.; Miyawaki, K.; Shinozuka, J.; Uetsuka, K.; Nakamura, S.-I.; Kimura, N.; Yoshikawa, Y.; Doi, K. Cerebral amyloid angiopathy in an aged great spotted woodpecker (Picoides major). Neurobiol. Aging 1999, 20 (1), 53-56.

(44) Coppola, V. J.; Kanyok, N.; Schreiber, A. J.; Flaim, M. E.; Bingman, V. P. Changes in hippocampal volume and neuron number co-occur with memory decline in old homing pigeons (Columba livia). Neurobiol. Learn. Mem. 2016, 131, 117-120. 